\title{
AN ANALYSIS OF ULTRASONIC WAVE PROPAGATION IN METALLIC PIPE STRUCTURES USING FINITE ELEMENT MODELLING TECHNIQUES
}

\author{
A. Gachagan $^{1}$, P. Reynolds ${ }^{2}$ and A. McNab ${ }^{1}$ \\ ${ }^{1}$ Centre for Ultrasonic Engineering, University of Strathclyde, Glasgow, UK; \\ ${ }^{2}$ Weidlinger Associates Inc, Los Altos, U.S.A
}

\begin{abstract}
This paper describes the development of a large finite element (FE) model representing ultrasonic inspection in a metallic pipe. The model was developed using PZFlex and comprises two wedge transducer components, water coupled onto the inner wall of a 36 inch diameter steel pipe. The $2 \mathrm{MHz}$ transducers are separated by $430 \mathrm{~mm}$ and configured to generate/receive ultrasonic shear waves. One device is used in pulse-echo mode to analyse any reflected components within the system, with the second transducer operating in a passive mode. Importantly, to minimise the models computational requirements, an external pressure loading function was applied to the wedge component within the model to simulate the transducer excitation. A number of simple defect representations have been incorporated into the model and both the reflected and transmitted ultrasonic wave components acquired at each wedge. Both regular slot and lamination defects have been investigated, at three different locations to evaluate the relationship between propagation path length and defect response. These defect responses are analysed in both the time and frequency domains. Moreover, the FE modelling has produced visual interpretation, in the form of a movie simulation, of the interaction between the propagating pressure wave and the defect. A combination of these visual aids and the predicted temporal/spectral waveforms has clearly demonstrated the essential differences in the response from either a slot or lamination defect.

Introduction: Ultrasonic wave propagation and its interaction with defects are of considerable interest to the NDE community. This paper describes the generation of a finite element (FE) model, using PZFlex, for investigating the generation and detection of shear waves within metallic pipe structures and their ability to detect axial cracks in gaseous pipeline environments. The system under investigation comprises a $60^{\circ}$ arc of the pipe, with two ultrasonic

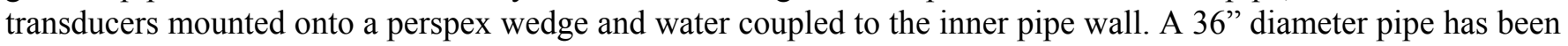
modelled with the transducer separation distance fixed at $430 \mathrm{~mm}$. One transducer is driven at an operating frequency of $2.1 \mathrm{MHz}$ to launch the shear wave energy into the pipe and the received temporal waveforms analysed at both transducer locations. Six modelled defects were introduced at three locations along the propagation path, corresponding to a total number of 18 simulation runs. The defect representations were categorised as either an outer wall breaking crack or a mid-plane lamination. The time waveforms for each simulation were collected at both transducer positions and analysed in both the time and frequency domains. This information, along with visual interpretation of the defect interaction, can then be used to identify defect discrimination criteria between the modelled crack and lamination type defects.
\end{abstract}

Methodology: This programme of work has utilised PZFlex, a finite element analysis tool [1-3], to simulate the generation, propagation and detection of elastic waves in a metallic pipe structure. A 2D model was developed in three stages: transducer interface incorporating excitation mechanism, angled wedge, water coupling and interface to load medium; curved pipe section incorporating two facing transducer configurations; and the introduction of defects. The overall model corresponds to a pipe length of approximately $0.5 \mathrm{~m}$, with a thickness of $9.3 \mathrm{~mm}$, and a transducer separation of $430 \mathrm{~mm}$. The operating frequency is $2.1 \mathrm{MHz}$, which corresponds to an operating frequency of approximately $1.5 \mathrm{~mm}$. Hence, it should be noted that this configuration corresponds to a minimum propagation path length in excess of 150 wavelengths. Consequently, it was necessary to incorporate denser meshing within the FE model and run the simulations on a multi-processor SGI computer facility to produce accurate results.

Transducer Interface Model: The transducer type used to collect data for this project is a commercial angle shear wave probe. This device operates at $2.1 \mathrm{MHz}$, is attached onto a perspex wedge and water coupled to the inner wall of the steel pipe. The transducer is mounted at an angle in the perspex wedge such that the ultrasonic energy incident on the surface of the sample under test will produce shear waves within the test material. This face of the wedge will be referred to as the contact face throughout this paper.

To simplify the model and provide an enhanced simulation runtime, the transducer response was simulated as a pressure loading onto the contact face of the Perspex wedge. For this study, plane wave excitation was modelled as an uniform, co-phased pressure load applied to the contact face of the transmitting wedge. Figure 1 
illustrates the simulated transducer interface. The angled wedge is water coupled to the steel pipe load and includes a rubber damping layer to minimise reverberations within the wedge.

Pipe Model Development: To complete the modelled configuration, a second perspex wedge arrangement was incorporated into the 2D finite element model. The distance between the transducers was $430 \mathrm{~mm}$, and is illustrated in Figure 2. The two wedges are positioned in the model facing each other. Thus the angled wedges will produce and accept shear waves energy propagating within the pipe wall. Absorbing material, an invented highly damped steel, has been placed at both ends of the pipe to minimise the reflection of ultrasonic energy from the discontinuities at the ends of the pipe.

Introduction of Defects: The final stage in the development of the NDE model was the introduction of a defect within the steel structure. The defect scenarios were chosen to represent simple crack and lamination type defects. The crack defects were all surface breaking on the outer wall of the pipe and had lengths of $1 \mathrm{~mm}, 3 \mathrm{~mm}$ and $6 \mathrm{~mm}$. The laminations type defects were located in the mid-plane of the pipe wall and were $10 \mathrm{~mm}$ and $5 \mathrm{~mm}$ in length. All defect types were $0.1 \mathrm{~mm}$ in width and represented as air in the model. The defects could be located at three positions between the two wedges, corresponding to $110 \mathrm{~mm}, 215 \mathrm{~mm}$ and $320 \mathrm{~mm}$ from the transmitting wedge. Figure 3 illustrates the $6 \mathrm{~mm}$ long crack on the outer surface of the pipe wall, at the $110 \mathrm{~mm}$ position. The graphical window representation chosen for this simulation illustrates three parts of the model, where the top and bottom windows depict the region around each wedge and the middle window offering a magnified view of the pipe with the defect incorporated. The temporal waveforms at both transducer sites were predicted for each of the modelled defect scenarios. Post-processing in the form of bandpass filtering and a FFT [4] were then applied to these waveforms. The processed temporal and spectral responses were subsequently evaluated for potential defect discrimination characteristics.

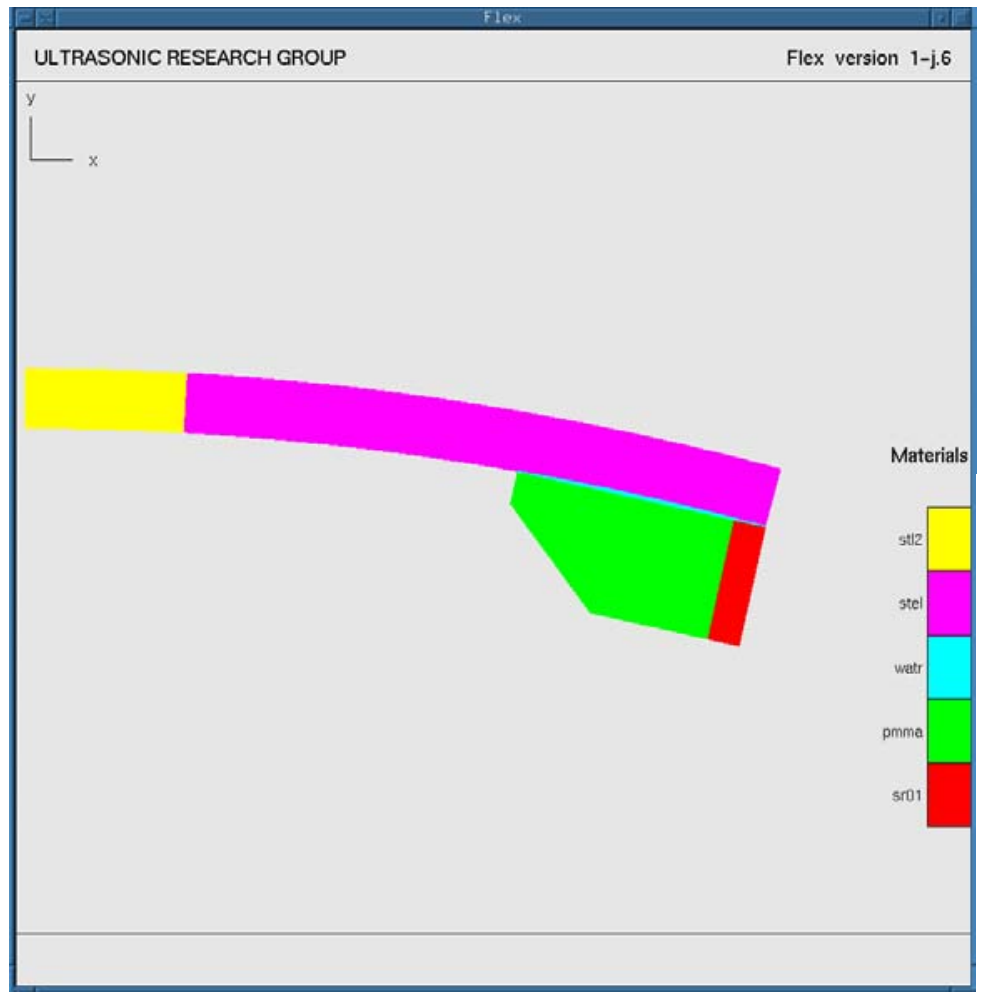

Figure 1
Model illustrating transducer interface
Note: Material mnemonics

stl2 - highly damped steel

stel - steel

watr - water

pmma - perspex

sr01 - rubber 


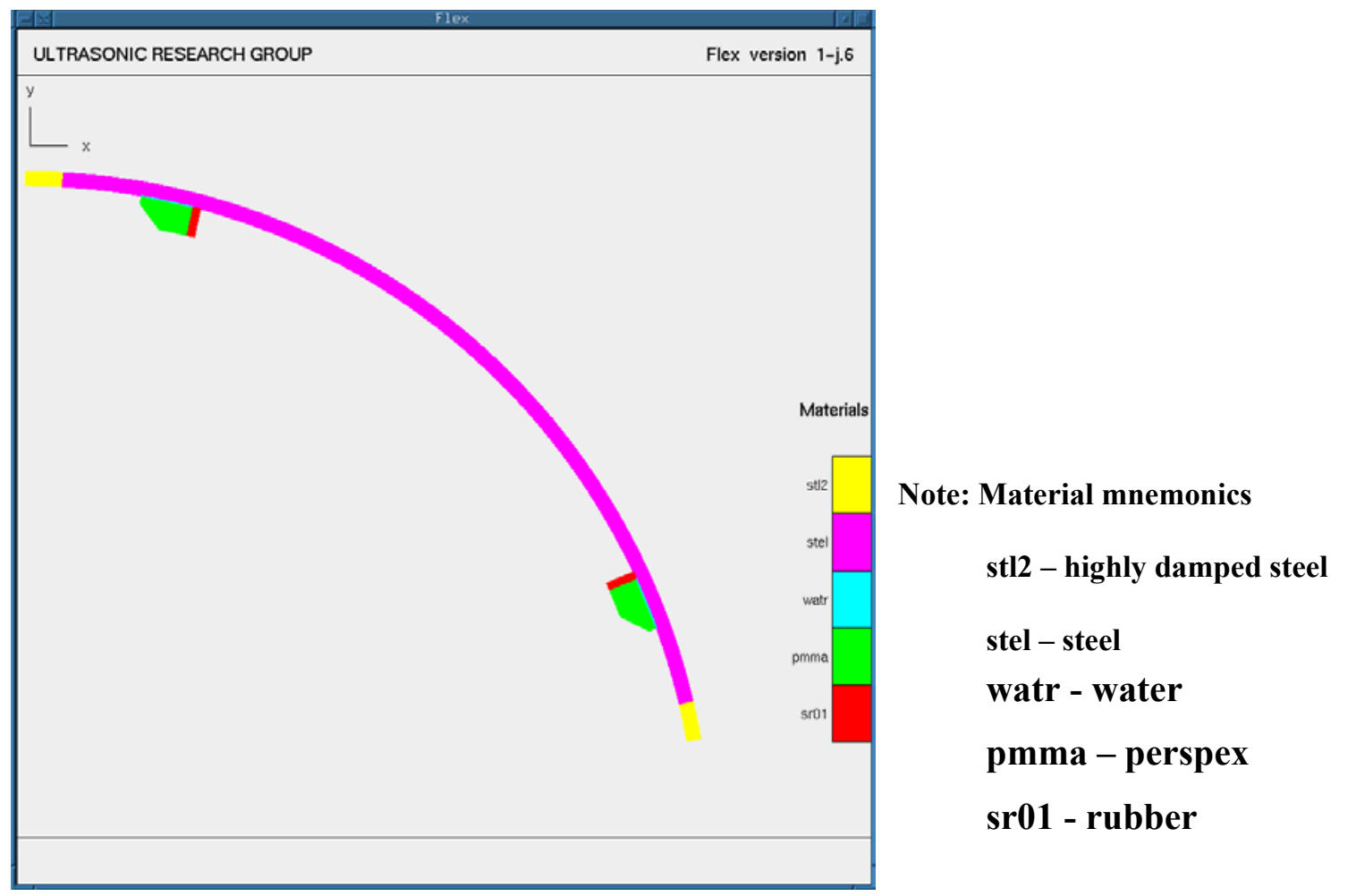

Figure 2 Full FE model incorporating steel pipe and two transducer wedge configurations 


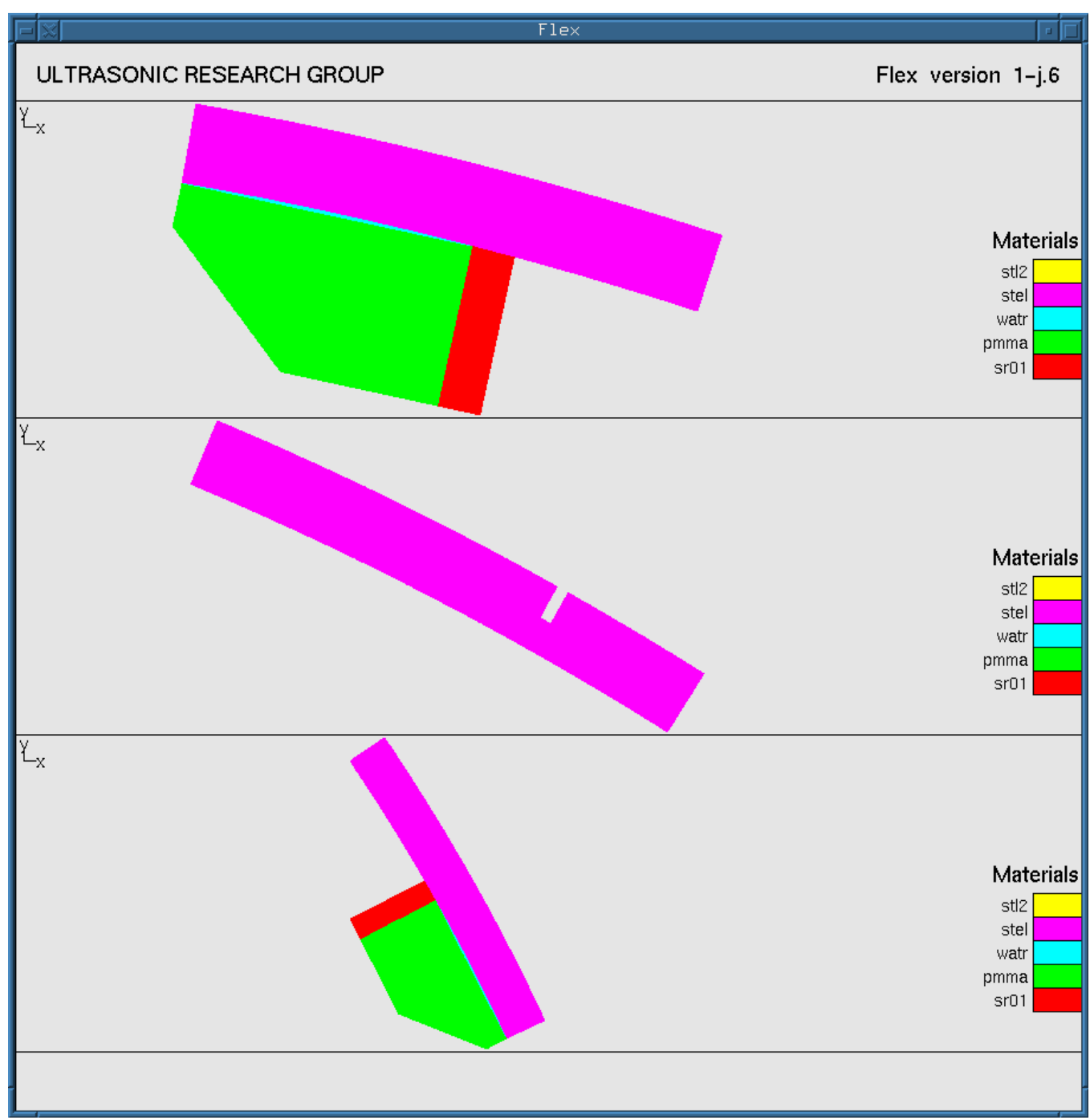

Figure $3 \quad$ Introduction of a crack defect into the FE pipe model

Results: With the full FE model developed, it was then utilised to provide predicted results for pulse-echo and pitch-catch propagation and to help visualise defect interaction with the propagating ultrasonic energy. In the following section, both time and frequency characteristics will be used to describe the defect interaction with sample crack and lamination type defects. It should be noted that the first $100 \mu$ s of the time data has been removed for clarity of signal interpretation. This is similar to the practical NDE instrumentation which include time-gating functionality.

Figures 4(a) and (b) illustrate the reflected and through transmission data after interaction with a 6mm slot, located $110 \mathrm{~mm}$ from the transmitting wedge. The magnitude of the reflected signal, Figure 4(a), is an order of magnitude above the temporal characteristic for the no defect situation. Correspondingly, the magnitude of the transmitted signal, Figure 4(b), has been significantly reduced from the no defect simulation result. This result was expected due to the fact that the defect spanned almost two thirds of the width of the pipe wall.

Figures 5(a) and (b) illustrate the reflected and through transmission data after interaction with the 5mm long lamination defect, again located at the $110 \mathrm{~mm}$ location. Interestingly, the reflected signal, Figure 5(a), is less defined than with the crack defect scenario presented in Figure 4(a). In addition, the frequency spectrum of the through transmitted signal, shown in Figure 5(b), illustrates a lack of $1.65 \mathrm{MHz}$ and $2.1 \mathrm{MHz}$ components, which relate to the most prominent components in the reflected data. 


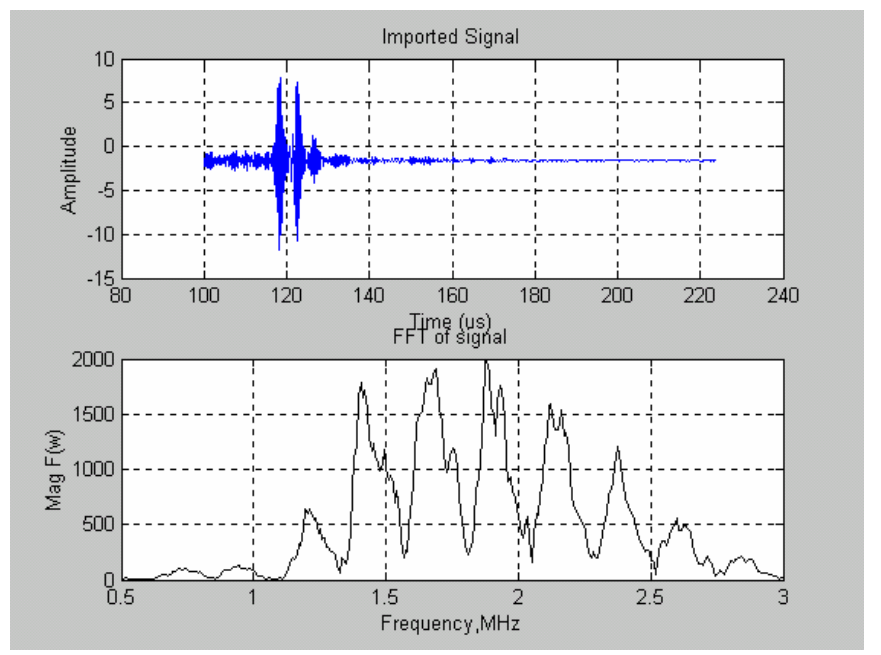

Figure 4(a) Reflected waveform from a $6 \mathrm{~mm}$ crack located at $110 \mathrm{~mm}$

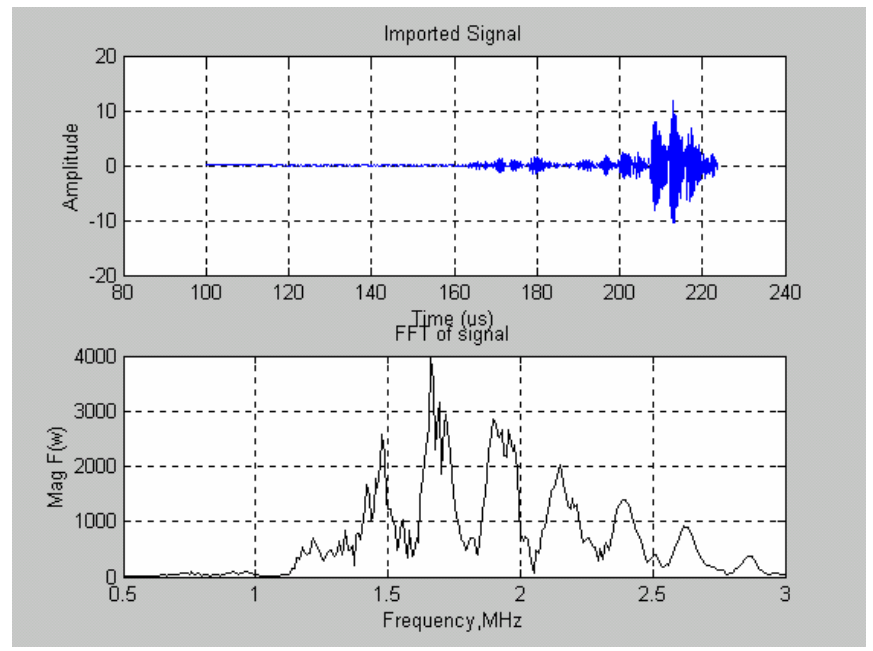

Figure 4(b) Transmitted waveform from a $6 \mathrm{~mm}$ crack located at $110 \mathrm{~mm}$

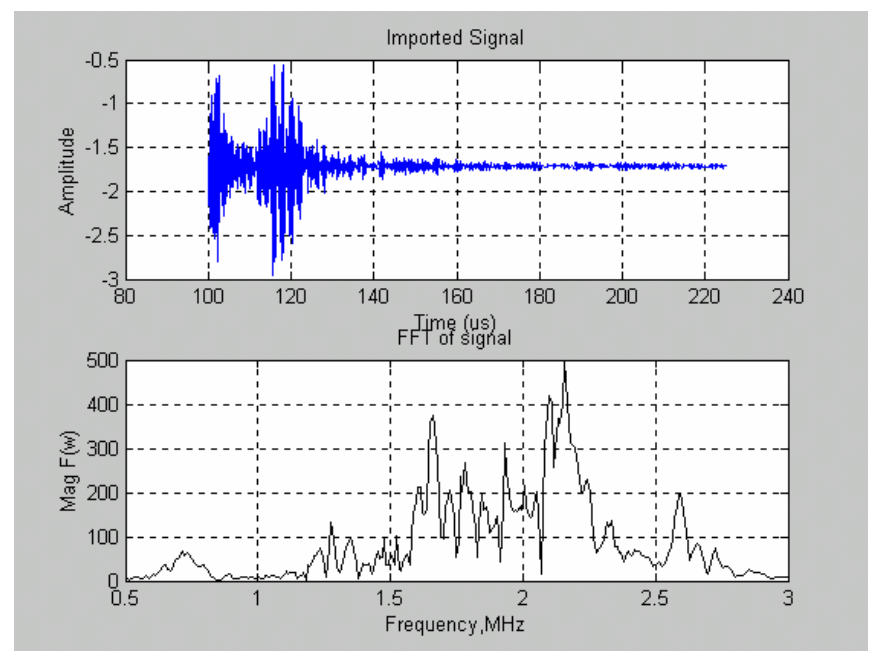

Figure 5(a) Reflected waveform from a $5 \mathrm{~mm}$ lamination located at $110 \mathrm{~mm}$ 


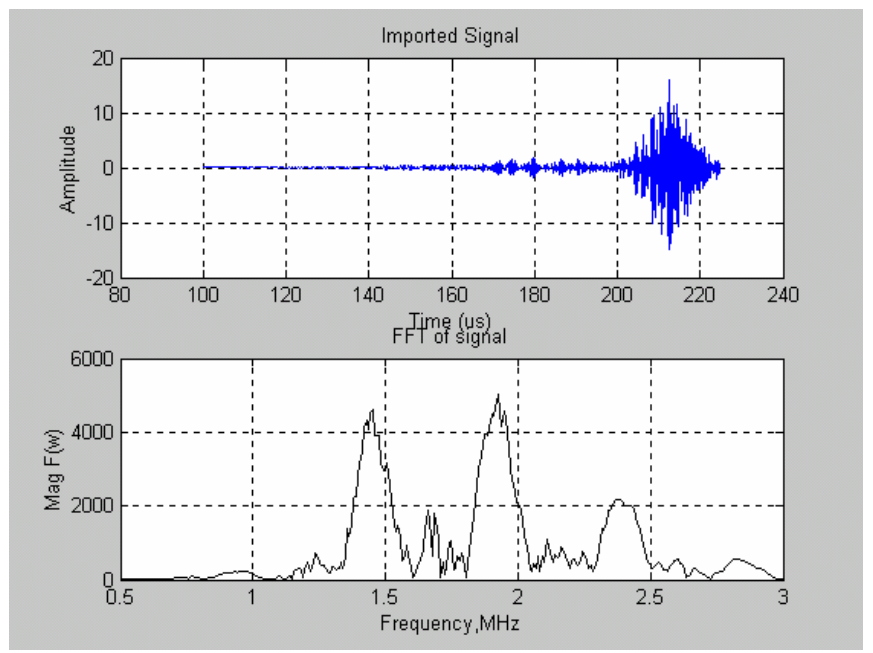

Figure 5(b) Transmitted waveform from a $5 \mathrm{~mm}$ lamination located at $110 \mathrm{~mm}$

Discussion: The defect scenarios were also analysed using the visualisation facility in the FE code. Each simulation produced a number of images depicting the pressure distribution around the defect location. These images were sequenced together to generate movies illustrating the interaction between the propagating ultrasound and the defects. In each simulation, the defect interaction with the propagating ultrasonic energy is evident. Interestingly, towards the end of the interaction, the defect can almost be considered as a secondary source of energy. This event occurs due to a surface wave propagating along the surface of the crack, or lamination, and results in ultrasonic energy radiating into the pipe, from one end of the defect, in a manner analogous to a spherical point source. Furthermore, for a slot defect this secondary energy is transmitted in both directions in the pipe, but for lamination type defects the energy is transmitted only down the pipe to the second wedge. Snapshots of this effect are presented in Figures 6(a) and 6(b) for the $6 \mathrm{~mm}$ slot, located at the $110 \mathrm{~mm}$ position, and the $5 \mathrm{~mm}$ lamination, located at $215 \mathrm{~mm}$ position.

Analysis of the temporal and frequency characteristics for the reflected and transmitted signals, for all defect scernarios, illustrate two potential discrimination techniques. The first technique is a straightforward threshold detector on the reflected waveform data. For the $3 \mathrm{~mm}$ and $6 \mathrm{~mm}$ crack simulations, the magnitude of the reflected data was a clear indicator of the presence of a significant crack. The reflection from the $1 \mathrm{~mm}$ crack was of a magnitude comparable to the lamination type defects and hence, an alternative approach must be adopted. Hence, the second technique is an analysis of the frequency spectrum from both the reflected and through transmitted ultrasonic waveforms. In the case of a lamination, the simulations clearly illustrate that the dominant spectral components of the reflected signal correspond to a low magnitude in the corresponding frequencies of the transmitted signal. Whereas, the spectral response from the crack type defects are more structured, with a series of distinct frequency components evident in both the reflected and transmitted frequency characteristics.

To summarise, the reflected temporal waveform must be used to give an indication of the presence of a defect in the pipe. This can be used to identify the location of the flaw with respect to the transmitting transducer position, using standard time-of-flight techniques. Subsequently, the frequency characteristics of both the reflected and transmitted signals should be analysed to determine the defect classification. A series of distinct frequency peaks in both reflected and transmitted responses indicates the presence of a crack type defect. Furthermore, an indication of the crack length could be determined from the magnitude of the reflected time data. Laminations can be characterised by frequency peaks in the reflected data directly corresponding to a low frequency magnitude region in the transmitted data. Potentially, these actual frequency components could directly relate to the length of the flaw. 


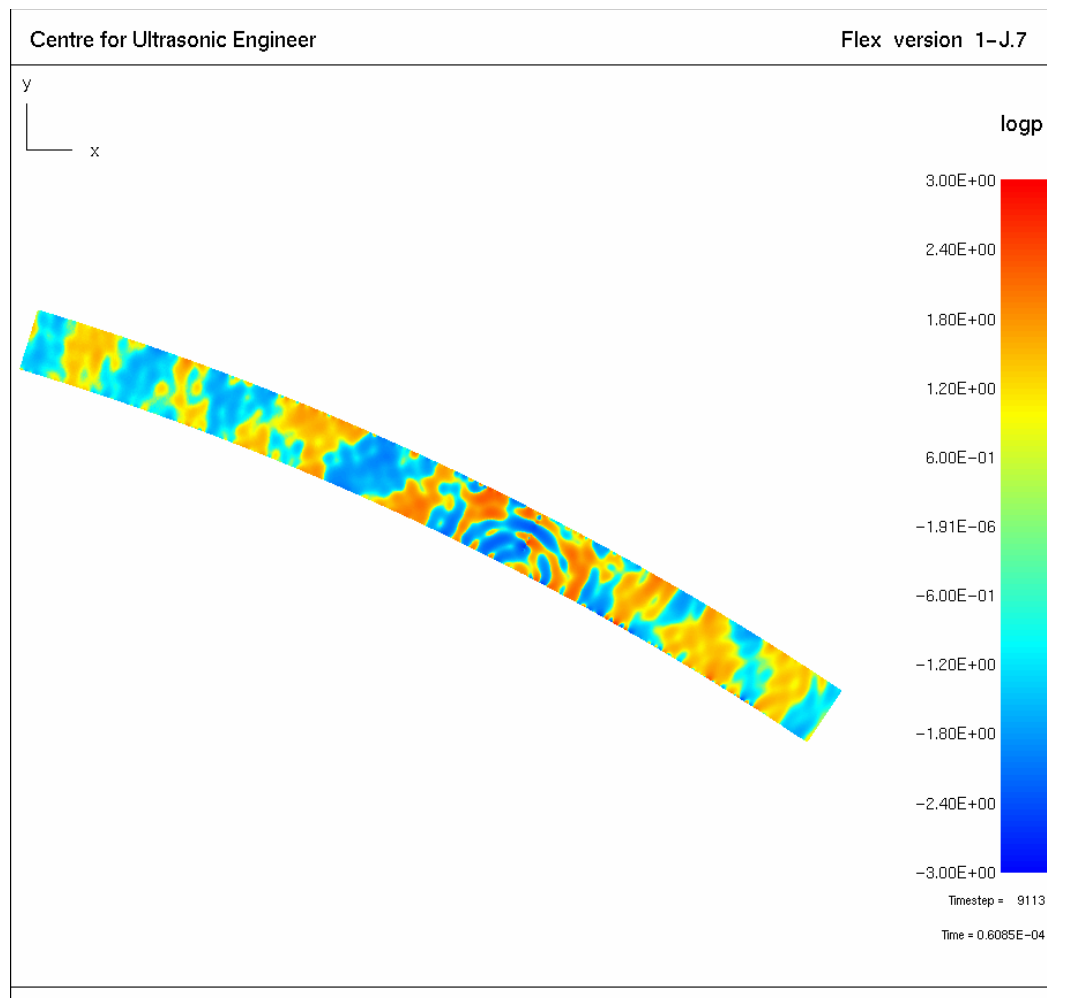

Figure 6(a) Tip of 6mm crack acting as a secondary source of ultrasonic energy

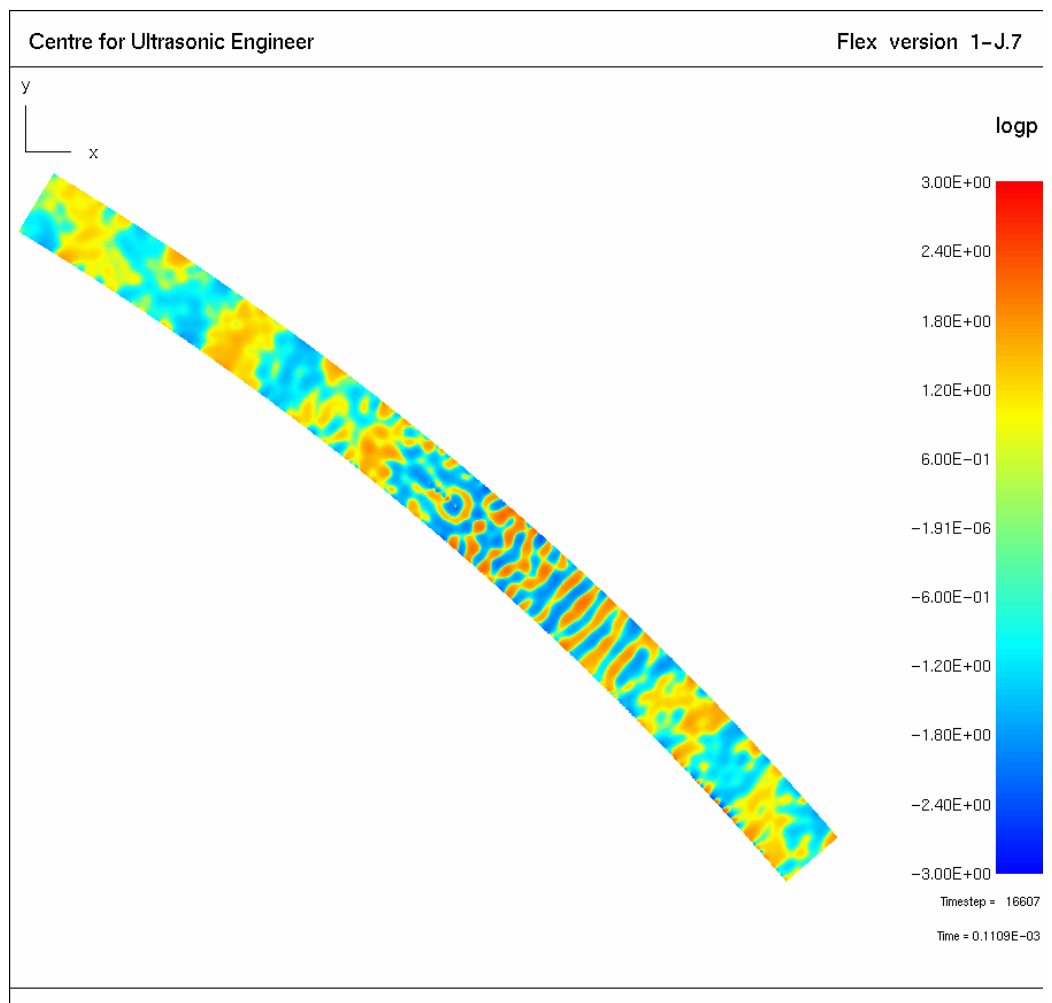

Figure 6(b) 5mm lamination acting as a secondary source of ultrasonic energy 
Conclusions: This paper has described a finite element representation of two shear wave transducers coupled onto a steel pipe. The pipe diameter is 36" and although only approximately $1 / 6$ of the pipe has been modelled, the 2D representation exceeds the recommended model size for typical FE simulations. In order to efficiently compute with such a large model, the FE representation is purely mechanical and the ultrasonic transducers are not incorporated into the model. For this work, the ultrasonic energy is induced into the pipe structure by applying a pressure loading function. Furthermore, a multi-processor facility has been used to reduce simulation runtimes. This work has pushed the boundaries for FE simulation of large scale models and within the current model, propagation path lengths in excess of 300 wavelengths have been analysed.

Analysis of the defect interaction with the propagating ultrasonic energy in the simulations has indicated that there are potential mechanisms to assist with the defect discrimination process. One of the most important conclusions from the programme of work is that both time and frequency characteristics are required from both the reflected and transmitted responses to identify the defect type. It is interesting to note that there is a difference in both the visualisation and data analysis for the crack and lamination type defects.

\section{References:}

1. PZFLEX User's manual, Version 1-J7, November 2002

2. Wojcik G, Vaughan D, Mould J, Powell D and Hyslop J, '3D Time-Domian Modelin for Broadband Transducer Array Design', Review of Progress in QNDE, 2001, pp 906-913

3. Mills DM and Smith SW, 'Finite Element Comparison of Single Crystal vs. Multi-Layer Composite Arrays for Medical Ultrasound', IEEE Trans UFFC, Vol 49(7), jul 2002, pp 1015-1020

4. Mould JC, Wojcik GL, Carcione LM, Tabei M, Mast TD and Waag RC, 'Validation of FFT-Based Algorithmsfor Large Scale Modeling of Wave propagation in Tissue', IEEE Ultrasonics Symposium, 1999, http://www.wai.com/AppliedScience/Software/Pzflex/index-pz.html 